\title{
TRINA MERCADER: UNA EXPERIENCIA DE CONVIVENCIA CULTURAL EN MARRUECOS
}

\author{
Fernando de Ágreda Burillo
}

Recordar a Trina Mercader - y a Eugenia Gálvez, por otra parte- es celebrar un homenaje lleno de nostalgia y, también, una obligación para sus amigos, tanto españoles como marroquíes.

Es cierto; se trata, además, de una obligación para los que tuvimos la oportunidad de tratar a Trina y gozar de su amistad, de su "fe ingenua e infantil", como decía Pío Gómez Nisa en la presentación de su primer libro de poemas ${ }^{1}$. Es una mujer "a la que la poesía española y marroquí debe un gran homenaje para que su nombre no caiga en el olvido", tal como afirmaba otro buen amigo de Trina, Jacinto López Gorgé, que aseguraba, voluntarioso: "no lo caerá. Estoy seguro. Porque, más pronto o más tarde, llegará ese merecido reconocimiento a su gran obra poética" ${ }^{2 "}$.

La huella de Marruecos en la vida y la obra de Trina Mercader nos la describía ella misma en su autopresentación de la citada obra Tiempo a salvo: "Mi primer nacimiento, en Alicante. El segundo, en Larache. Mi biografía debería titularse "Historia de una revista". Porque una revista -Al-Motamid- es la que centra y orienta mi vida en Marruecos". Y subrayaba tántos hallazgos de aquella experiencia: los poetas marroquíes y las versiones bilingües de nuestra poesía; hallazgo de los más jóvenes arabistas españoles...". "El proyecto -diría la propia Trinase lleva a cabo con una pobreza de medios que contrasta con la ambición que lo mueve ${ }^{3 "}$.

Afortunadamente, todavía existen amigos de Trina, más autorizados por tanto, que se han referido a su obra tras la vivencia de aquellos años: Pedro Martínez Montávez, del que Trina publicó en el año 1956 el libro La Escuela siro-americana, dentro de la colección "Itimad", aneja a la revista, y que pronunció una conferencia en la Sala de Cultura Española de esta ciudad, en 1965, que llevaba por título: "Notas sobre el tema árabe en la poesía española actual”. Al referirse a Marruecos, a las revistas de poesía y a sus autores, decía:

1 Tiempo a salvo.Tetuán, colección "Itimad, n" 3. 1956.

2 "Dos revistas hispano marroquíes", publicado en Encuentros literarios: Marruecos-España-Iberoamérica. Volumen preparado por Mohammad Chakor. Madrid, 1987.

3 "Al-Motamid e Itimad: una experiencia de convivencia cultural en Marruecos", por Trina Mercader. En Revista de Información Cultural de la Comisión Nacional Española de Cooperación con la UNESCO. N² 25, enero-marzo, 1981 , p. 76. 
"Sin embargo, a mí me parece representativo de los afanes y las luchas de todos el nombre de Trina Mercader. Por lo que tiene de pasión femenina, por lo que tiene de adelantado, por lo que tiene de entrega apasionada a esta tierra en que vivió. Por lo que significó también su labor común de trabajo con los jóvenes poetas marroquíes ..."

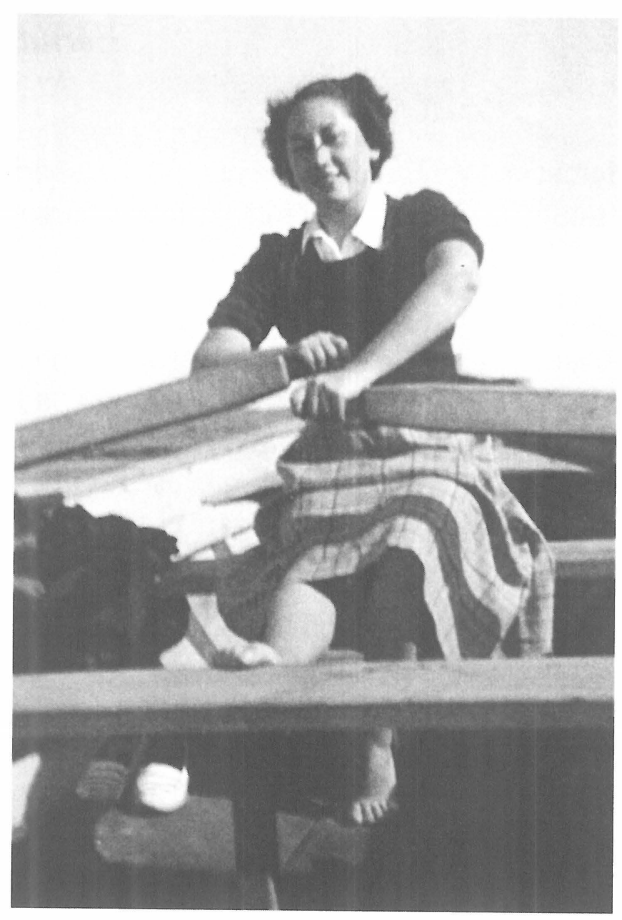

Trina Mercader. Larache, 1951. Propiedad de D. Fernando Díaz Esteban

Jacinto López Gorgé colaboró activamente con Trina formando parte del Consejo de redacción de su revista y, además, conocería la experiencia de dirigir otra publicación, también en Tetuán, cuya línea fue, en cierto modo, continuación de Al-Motamid. Me refiero a Ketama, suplemento literario de Tamuda, la conocida revista de investigación que se publicó de 1953

\footnotetext{
${ }^{4}$ Cfr. Cuadernos de la Biblioteca Española de Tetuán, n 3, junio, 1966. Esta revista fue dirigida, desde 1977 hasta su desaparición, por otro buen amigo nuestro: Guillermo Gozalbes Busto, quien, como Trina, se trasladó a Granada, donde residió hasta su fallecimiento. Guillermo me facilitó la dirección de Trina en aquella ciudad. Nunca olvidaré su gran humanidad.
} 
a $1959^{5}$. Jacinto ha escrito, como es sabido, numerosos artículos sobre aquellas revistas ${ }^{6} \mathrm{y}$, al referirse a Al-Motamid, nos ha hablado de Trina, de su labor en Marruecos y del grupo de poetas -de ambas orillas- que participaron en su andadura. En 1991 él mismo nos ofrecía una muestra de su activa labor de antólogo en el libro Marruecos en la poesía española contemporánea, publicado en Granada, dentro de la colección "Ibermagrib". En la presentación del mismo-jtantos autores que tan bien conoce, en un tema tan próximo!"'- hace una extensa referencia a nuestra autora y acaba reclamando la anunciada publicación de los escritos inéditos de Trina. Recordemos sus propias palabras:

"Pero Trina Mercader, además de lo que publicó en vida, en Marruecos y en España, dejó mucha poesía inédita, y no poca de tema marroquí. Material más que suficiente para otro volumen de la colección "Ibermagrib", que brindó al poeta Antonio Carvajal, depositario de todo ello como heredero del archivo literario de Trina, con el que estoy dispuesto a colaborar incondicionalmente".

En plenos años cincuenta -de 1953 a 1954, concretamente, y gracias al impulso de $\mathrm{Al}$ Motamid- se iniciaría una antología de grandes poetas contemporáneos de la mano, primero, del escritor chileno, de origen sirio, Benedicto Chuaqui. Trina Mercader había dado a conocer su obra, y, a solicitud de ella, Chuaqui envió el artículo titulado "Poesía árabe contemporánea”, que se publicaría en el número 25 , correspondiente a marzo de $1953^{7}$.

Pero la difusión más amplia de la poesía árabe llegaría de la mano del conocido escritor tetuaní Muhammad al-Ṣabbāg. De él ha dicho López Gorgé: "La gran labor de Muhammad Sabbag por aquel tiempo, multiplicándose por atender a dos revistas -Al-Motamid y Ketamano es fácil de olvidar, por muchos años que hayan transcurrido. Él es quien fue acercándonos, de una manera global y pormenorizada, a la gran poesía árabe del novecientos, escasamente conocida en España"8. Los contactos de al-Șabbāg con los escritores orientales-libaneses especialmente hicieron familiares nombres tan destacados como los de Yuubrān Jalī Ŷubrān, Mijā'îl Nucayma, Būlus Salāma, Sa ${ }^{\complement} \overline{1}{ }^{~}{ }^{c} A q 1$, Fadwà Ṭūqān, etc.

5 Desde 1959, Tamuda dejaría de publicarse en Tetuán, para unirse a Hesperis, su homóloga en la antigua zona francesa.

${ }^{6}$ Ha pronunciado, asimismo, diversas conferencias sobre el mismo tema: en 1985, concretamente, participó en el "Aula de Poesía en memoria de Trina Mercader", celebrado en el Palacio de la Madraza, de Granada. Además, se celebró una lectura de poemas -algunos inéditos-, en la que intervinieron:Elena Martín Vivaldi, Eulalia Dolores de la Higuera, $\mathbf{M}^{\mathbf{a}}$ del Carmen Pérez Vera, Rafael Guillén, Carlos Villareal y Antonio Carvajal.

7 Matías Rafide da noticias de Chuaqui en su libro Escritores chilenos de origen árabe, editado por el Instituto Chileno-Árabe de Cultura en 1989. Otro miembro del grupo de traductores de Al-Motamid era Dris Diuri, al que Trina conoció en la Municipalidad de Larache. Mohammad Chakor y Sergio Macías se refieren a esta significativa y casi ignorada figura del hispanismo marroquí en su artículo "Literatura marroquí en lengua castellana", incluido en el libro ya citado Encuentros literarios: Marruecos-España-Iberoamérica, p. 14 y ss. Su hijo "Adelilah me ha comunicado amablemente que Dris Diuri redactó unas memorias en las que recogía los recuerdos de aquella época. Según parece, y lamentablemente, problemas familiares le han impedido tener acceso al manuscrito que dejó su padre.

${ }^{8}$ Cfr. Nota 2. 
Esta labor se vería continuada por el mismo al-Șabbāg en las páginas de Ketama, tal como ha subrayado López Gorgé en el prólogo a la antología dedicada a su gran amigo, Del fuego $y$ de la luna y otros poemas, publicada en la colección “Adonais", en 1990.

Trina Mercader vino a Madrid, en mayo de 1980, invitada por el Instituto Hispano-Árabe de Cultura para hablarnos de sus vivencias en Marruecos $y$, especialmente, de sus publicaciones y del significado de las mismas. El título de su conferencia fue precisamente "Al-Motamid e Itimad: una experiencia de convivencia cultural en Marruecos". Un resumen de la misma, lo principal de su contenido, se publicó en la Revista de Información de la Comisión Española de Cooperación con la UNESCO, número 25, enero-marzo de 1981, que dirigía entonces Fernando Valderrama Martínez, otro de sus antiguos amigos de Tetuán y figura muy conocida en esta ciudad, donde trabajó durante muchos años.

En aquel viaje a Madrid Trina venía acompañada de sus primos de Alicante: María y Bonifacio Balaguer. Ellos, tras la muerte de Trina el 18 de abril de 1984, tuvieron la amabilidad de ampliar las noticias que ella misma me proporcionó. Así pude saber de su infancia en Torrevieja, donde se trasladó con su madre tras el fallecimiento de su padre; de su marcha a Larache, en 1936, donde ya residían sus primos. Allí viviría Trina, obligada por las trágicas circunstancias políticas, y llegaría a preparar las oposiciones para ingresar en la municipalidad. Tras un breve traslado a la antigua Villa Sanjurjo, en 1951, pasó a vivir a Tetuán donde se quedaría hasta su definitivo traslado a Granada en 1958.

Todavía conservo la relación de amistades que ella nos envió al Instituto, titulado entonces Instituto Hispano-Árabe de Cultura, para que diéramos aviso de su conferencia. Los primeros nombres correspondían a dos escritores que tanta importancia tuvieron en la historia de su revista, Carmen Conde y Vicente Aleixandre - ¡qué recuerdos de la antigua calle "Wellingtonia"!-. El tercero era ya el de una amiga marroquí, la conocida pintora Meriem Mizziam...

El 3 de mayo de 1984 Jacinto López Gorgé hablaría, también en el ciclo de conferencias que organizaba el tantas veces citado Instituto Hispano-Árabe de Cultura, de Trina Mercader, que acababa de fallecer. Jacinto rememoró aquel inesperado homenaje en su emocionado "Recuerdo a Trina Mercader", que publicó la revista Cálamo, número 2, julio-septiembre de 1984, con estas palabras:

"Hace muy pocas semanas, cuando me disponía a pronunciar una conferencia en el Instituto Hispano-Árabe de Cultura, me llamaron de Granada para decirme que Trina Mercader, la gran poetisa que fundó y dirigió durante nueve años la revista "AlMotamid", había muerto en el Hospital Clínico granadino. Al iniciar mi conferencia advertí al auditorio que les hablaba bajo la impresión de una muerte inmediata: una muerte que no podía apartar de mí ..."9.

\footnotetext{
${ }^{9}$ En aquella ocasión contamos, además, con la voz de la propia Trina grabada en cassette -recitando algunos de sus versos-, que nos ofreció, inesperadamente, su buena amiga Estrella Pérez de Amar.
} 
Hoy quisiera recordar, como modesto homenaje a nuestra amiga Trina Mercader, algunas de las cartas que me dirigió desde nuestra primera comunicación epistolar, en 1976, hasta finales de 1983. En aquel tiempo la enfermedad que le aquejaba estaba ya, desgraciadamente, muy avanzada.

En la primera, fechada el 2 de marzo de 1976, decía:

"Me interesa, así mismo, conseguir un catálogo o referencias de los escritores, pintores, dibujantes, escultores, ensayistas, historiadores y arqueólogos que hicieron obra sobre Marruecos, pues todo anda tan diseminado que no hay forma de recopilar de alguna manera un conocimiento del Marruecos de hace unos años, desde lo español".

Y en otra muy posterior, de 9 de mayo de 1983:

"El matrimonio Albarracín ${ }^{10}$ me llevó un domingo al Sanatorio, para que lo viera, la edición de un lujoso libro editado en Madrid recopilando todos los trabajos que habíamos hecho los españoles en Marruecos ..."

Se refería Trina al meritorio trabajo de Rodolfo Gil Grimau, titulado Aproximación a una Bibliografía española sobre el Norte de África (1850-1980), que, patrocinado por el entonces Embajador de España en Rabat, Alfonso de la Serna, editó el Ministerio de Asuntos Exteriores, a través de la Dirección General de Relaciones Culturales.

En 1985 se publica en Granada la Antología de relatos marroquíes en lengua española, primer volumen de la colección "Ibermagrib" recopilada por Mohammad Chakor y Jacinto López Gorgé. Tras un sugestivo prólogo de Antonio Gala, un fragmento del texto del Embajador y conocido hispanista "Abdul-Latif Jatib titulado "Un patrimonio común" (publicado inicialmente en Ketama y en el que defiende el papel de las revistas bilingües que, entonces, se publicaban), López Gorgé firma la "Justificación" del libro y recuerda, una vez más, a Trina Mercader con estas palabras:

"Sabemos cuánto le hubiera complacido este libro donde se recogen algunos de los cuentos a los que ella dio cabida en su revista de poesía, aunque Al-Motamid era fundamentalmente revista de poesía, al igual que su creadora, que fundamentalmente era poeta. Pese a que también cultivaba la prosa poética, y dedicó muchos poemas en prosa -y también en verso- a la temática marroquí, no dejó escrito, que nosotros sepamos, ningún cuento..."

Hoy, y con motivo de este recuerdo a la memoria de Trina, puedo decir que, entre los escritos inéditos que dejó, sí figuraban unos relatos de los que me habló en sus cartas con

\footnotetext{
${ }^{10}$ Gracias, también, a aquella correspondencia entré en contacto con Juan Martínez Ruiz y su mujer, Joaquina Albarracín. Destacadas figuras de los estudios marroquíes (él, a través del hispanismo; falleció en el año 2000) conocerían a Trina durante su estancia en Marruecos. Sobre su obra, cfr. Estudios dedicados al Profesor Juan Martínez Ruiz. Universidad de Granada, 1991.
} 
cierta frecuencia. Así, el 3 de mayo de 1979, decía: "Le adjunto uno de mis relatos, con dibujo mío, perteneciente a los relatos marroquíes que directamente tomé de Marruecos. Es Vd. el primero en conocerlo. El mismo no tiene más valor que la sinceridad y el cariño con que está escrito. El dibujo es del natural, de mis paseos por el barrio moro... Yo tenía proyectado editarlos por mi cuenta, con estos dibujos, en un formato pequeño de libro de bolsillo, primoroso. ¡Tanto cariño les tengo!..." Y proseguía, el 15 de febrero de 1981: "Estos días , pasado el frío de enero, he vuelto a revisar mis relatos marroquíes. Voy a recopilarlos con sus dibujos y darlos por terminados. Me entusiasmo con ellos. Lástima que no sean más numerosos...".

Para terminar, voy a leer aquel relato a que hacía referencia Trina, y que guardo con el mayor de los cariños, en espera de que algún día podamos verlos publicados, todos ellos, cumpliendo una de sus voluntades más queridas.

\title{
"UNA CALLE DEL BARRIO MORO DE LARACHE"
}

\author{
por Trina Mercader ${ }^{11}$
}

"Penetrar por una calle de Marruecos es abrir el libro de lo maravilloso. La luz vendrá, atravesando bóvedas, a nuestro encuentro. Porque hay que perderse, sin prisas, por el pequeño laberinto lumínico.

El barrio moro de Larache es ese laberinto de luces y sombras por donde me pierdo. Hay que aceptar la cuesta, y el guijarro resbaladizo, y la escalinata desigual y el rincón lóbrego y maloliente. Porque todo forma parte de esta escenografía ya en desuso en nuestro mundo civilizado, que nos engulle y atropella. Aquí, por el contrario, todo está a la mano, todo tiene una altura que no sobrepasa nuestra humanidad.

La misma estrechez de la calle es agradable a nuestra estatura. Es como andar por el interior de una casa grande, familiar. La voz del mendigo ciego nos acompaña desde todos los ángulos, resonando. La salmodia del almuédano, desde su torre, es una impresión nueva a nuestros oídos. La novedad, la sorpresa nos van acompañando. Los ojos se acostumbran a la luz y a la sombra simultáneas. La cal de las paredes tiene sólo la estridencia de la luz, el propio reflejo trascendido. Mi paso se hace lento, obligadamente parsimonioso. Aquí la prisa lo rompería todo.

Una mujer atraviesa la calle. El sol estalla en el blanco jaique y casi la transparenta. Los pliegues del manto retienen la sombra precisa, dándoles profundidad. Es un manto que tiene mucho de griego, en su cascada de pliegues a la espalda. De él emergen unos pies calzados de babuchas, blancas también, a ras de manto. Arriba, unos ojos negros, a veces verdes, en lo alto del letam, del velo. Acaso la tersura de una mejilla no vista, adivinada. El paso siempre

\footnotetext{
${ }^{11}$ Este relato se publicó en la revista Turia, de Teruel, $\mathrm{n}^{\circ}$ 37, junio de 1996, págs. 66-68, gracias a la amabilidad de Ana $\mathrm{M}^{\mathrm{a}}$ Navales, directora de dicha revista. Al enviárselo, pensé que a Trina le habría gustado verlo publicado, tal como me lo envió, aunque fuera solo y sin la introducción que hago ahora. Antonio Carvajal, conocido poeta granadino, a quien Trina dejó en herencia sus libros y escritos inéditos, ha contestado recientemente a las pesquisas realizadas con el propósito de cumplir la voluntad de su autora.
} 
es lento, comedido, remontando sin prisa la ascensión. La calle, las paredes de las casas son el marco de esa figura única, el único detalle vivo que aprisionan. La más leve esquina, una línea blanca entre lo blanco, la oculta, la desaparece. La calle, ahora, queda estática, más quieta que nunca, como en reposo.

Alguna puerta se entreabre. Un bisbiseo apenas perceptible, comenta en árabe: "Es una nazarena". Y la puerta se cierra blandamente, sin ruido, como la voz de las mujeres en el interior de la vivienda, o como sus pasos de pie descalzo sobre la cal de las azoteas.

En el recuadro blanco de otra azotea, una mujer se asoma:

- Buenos días, dice. Y sonríe.

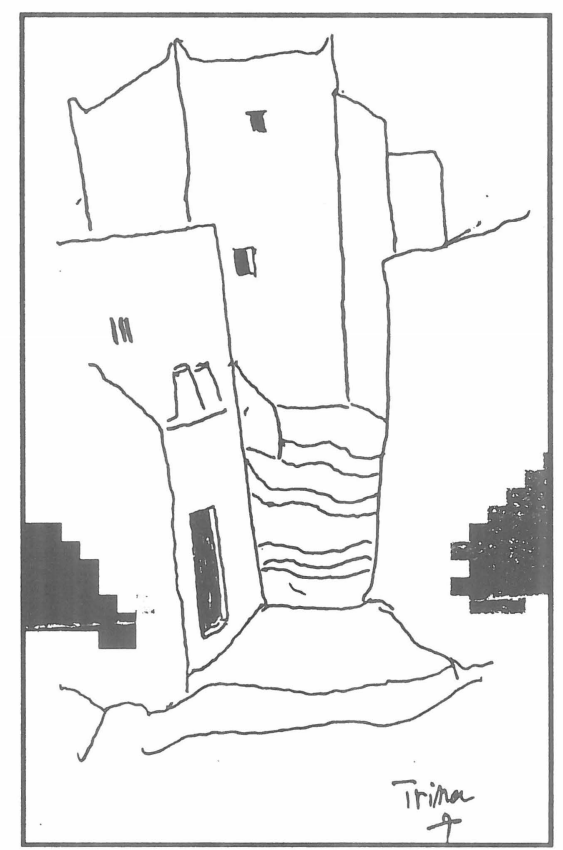

Es una mujer que quiere conversación. Es la clásica mujer de siempre, atenta a cualquier posibilidad de charla. La voz del ciego insiste, se alza o se pierde, para regresar una vez más, llenando las callejuelas con su eco. De pronto tropiezo con él, a bocajarro, en una esquina. Con su cayado tantea los pequeños peldaños. Me hago a un lado y le dejo pasar, mientras inicia una vez más su petición de ayuda.

Toda la calle asciende con mi propia ascensión. Su soberbia sube o baja su propio desnivel. Los edificios son enjutos, sobrios, de pequeñas ventanas altas que coronan las 
desiguales alturas. No hay tejados; solo una terminación brusca del blanco, cortando en cubos una arquitectura sin complicaciones.

A mi lado pasan los jaiques, las severas chilabas, destacando en lo blanco el amarillo limón de las babuchas. Los seres van como envueltos en su blancura. La calma de sus ademanes convierte cada calle en un claustro de mínimas proporciones. Claustro o celda para un pueblo religioso, en el que el silencio tiene una dimensión casi mística".

\section{BIBLIOGRAFÍA}

\section{AÑOS 40:}

- GARCÍA GÓMEZ, Emilio: Poemas arábigoandaluces. Madrid, Espasa Calpe. Colección Austral, $\mathrm{n}^{\circ}$ 162. 1940.

- GARCÍA GÓMEZ, Emilio: Biografías y estudios. Cinco poetas musulmanes. Madrid, Espasa Calpe. Colección Austral, n 513. 1944.

- SCHACK, Adolfo Federico de: Poesía y arte de los árabes en España y Sicilia. Traducción de Juan Valera. México, Ed. Centauro, S.A. 1944

\section{AÑOS 50:}

- PINILLOS, Manuel: "Literatura marroquí. Hacia una poesía de Marruecos. Noticias y comentarios sobre el tema”. En África, Año VII, n. 105, 1950, pp. 14-16.

- GIL BENUMEYA, Rodolfo: Hispanidad y arabidad. Madrid, Ediciones de Cultura Hispánica, 1952.

- GARCÍA GÓMEZ, Emilio: Poesía árabigoandaluza. Breve síntesis histórica. Madrid. Instituto Faruk I de Estudios Islámicos, 1952.

- RODRÍGUEZ JOULIA, Carlos: "Diez minutos con Trina Mercader y Dora Bacaioca, ganadoras del Premio "Marruecos 1953". En Diario de África, Tetuán, 23 de abril de 1953, pp. 14-15.

- MUHAMMAD IBN 'AZZUZ HAKIM: "Literatos modernos de Marruecos”. En África, 133, enero, 1953, pp. 14-15.

- MOHAMMAD SABBAG: El árbol de fuego. Versión del autor y de Trina Mercader. Colección Itimad, nº 1. Ediciones Al-Motamid, Tetuán, 1954.

- VALENTE, José Ángel: "Poesía árabe de hoy en Marruecos". En Índice, Madrid, septiembre 1955, p. 28. 
- GUASTAVINO GALLENT, Guillermo: De ambos lados del estrecho (Estudios breves hispano-africanos). Instituto General Franco de Estudios e Investigación Hispanoárabe. Tetuán.

- CONDE, Carmen: Empezando la vida (Memorias de una infancia en Marruecos). Colección Itimad, n² 2. Ediciones Al-Motamid, Tetuán, 1955.

- MERCADER, Trina: Tiempo a salvo. Colección Itimad, n 3. Ediciones Al-Motamid. Tetuán, 1956.

- MARTÍNEZ MONTÁVEZ, Pedro: La escuela siro-americana. Colección Itimad, n 4. Ediciones Al-Motamid, Tetuán, 1956.

- La Estafeta Literaria, n 94, 2ª́poca, Madrid, 4 de mayo de 1957: “Vida y aventura de las revistas de poesía". Encuesta entre varios directores de las mismas: Concha Lagos, Antonio y Carlos Murciano, Trina Mercader, José L. Estrada, Jacinto López Gorgé, etc ...).

- MARTÍNEZ MONTÁVEZ, Pedro: Poesía árabe contemporánea, Ed. Escélicer, Madrid, 1958.

\section{AÑOS 60:}

- LÓPEZ GORGÉ, Jacinto: "Las revistas, los periódicos, las hojas volanderas. Revistas poéticas”. En La Estafeta Literaria, nº extra, $2^{\text {a }}$ parte, enero 1964, pp. 282-283.

- MARTÍNEZ MONTÁVEZ, Pedro: "Notas sobre el tema árabe en la poesía actual". En Cuadernos de la Biblioteca Española de Tetuán, n 3, junio 1966, pp. 13-16, especialmente. Incluido, asimismo, en el libro del mismo autor: Ensayos marginales de arabismo. Universidad Autónoma de Madrid, Instituto de Estudios Orientales y Africanos, Madrid, 1977.

- VARIOS AUTORES: Contribución para una bibliografía de la literatura árabe del siglo XX. Cuadernos del Seminario de Pensamiento Árabe Contemporáneo del Instituto Hispano-Árabe de Cultura, nº 1, Madrid, 1966.

\section{AÑOS 70:}

- MARTÍNEZ MONTÁVEZ, Pedro: Introducción a la literatura árabe moderna. Madrid. Edición de la revistá Almenara, Madrid, 1974. De esta obra se ha publicado una segunda edición en la editorial CantArabia de Madrid, en 1985.

- RUBIO, Fanny: Revistas poéticas españolas, 1939-1975. Ed. Turner, Madrid, 1976 (Tesis doctoral). 
- ÁGREDA BURILLO, Fernando de: "Datos sobre las traducciones al árabe de la poesía española. La revista Al-Motamid”. Revista del Instituto Egipcio de Estudios Islámicos en Madrid, volumen XIX, 1976-1978, pp. 115-125.

- LÓPEZ GORGÉ, Jacinto: “Vicente Aleixandre y sus cartas a revistas jóvenes de poesía (1948-1958". La Estafeta Literaria, nº 625 (1 de diciembre de 1977), pp. 15-17.

\section{AÑOS 80:}

- MARTÍNEZ MONTÁVEZ, Pedro: "Las relaciones literarias hispano-árabes contemporáneas. Planteamiento de la cuestión". En Estudios de Asia y África, El Colegio de México, 43, enero-marzo, 1980, pp. 102-123, y recogidos en Literatura árabe de hoy, del mismo autor, Ed. CantArabia, serie estudios n 4, Madrid, 1990, pp. 15-35.

- MERCADER, Trina: "Al-Motamid e Itimad: una experiencia de convivencia cultural en Marruecos". En Revista de Información de la Comisión Nacional Española de Cooperación con la UNESCO, Madrid, n² 25, enero-marzo 1981, pp. 76-80.

- VARIOS AUTORES: Literatura y pensamiento marroquíes contemporáneos. Instituto Hispano-Árabe de Cultura. Facultad de Letras de Rabat. Seminario de Literatura y Pensamientos Árabes Modernos. Serie “Antologías Nacionales III”, Madrid, 1981.

- GIL GRIMAU, Rodolfo: Aproximación a una bibliografía española sobre el Norte de África. Ministerio de Asuntos Exteriores. Dirección General de Relaciones Culturales, Madrid, 1982.

- LÓPEZ GORGÉ, Jacinto: "Recuerdo a Trina Mercader". En Cálamo, Instituto HispanoÁrabe de Cultura, 1984, nº 2, julio-septiembre, p. 45.

- LÓPEZ GORGÉ, Jacinto: “Al-šicr al-carabī wa-l-isbānī fī rihlat al-tawassul”. Trad. de Ahmad al-Mațlūb. Rabat, Al-'Alam al-Taqā̃fī, nº 704, 4 de agosto de 1984.

- CARVAJAL, Antonio: "Trina Mercader ha dejado una importante obra inédita". Diario de Granada, 18 de abril de 1985.

- FERNÁNDEZ, Miguel: “Trina Mercader. In Memoriam”. En Melilla Hoy, 10 de mayo de 1985.

- CIGÜEÑA BECCARIA, María Dolores: Bibliografía de D. Emilio García Gómez. Memoria de Licenciatura (Inédita). Facultad de Filología, Universidad Complutense. Sección de Filología Hispánica. Subsección de Literatura Hispánica, Madrid, 1985.

- MOHAMMAD CHAKOR Y LÓPEZ GORGÉ, Jacinto: Antología de relatos marroquíes en lengua española. Ed. Ubago, Colección Ibermagrib, Granada, 1985. 
- LÓPEZ GORGÉ, Jacinto: “Ante la tumba del Rey-Poeta Al Mutamid Ibn Abbad, con Trina Mercader en el recuerdo". En Homenaje poético a Al-Mutamid, Universidad de Verano Al-Muctamid Ibn Abbad. Assilah (Marruecos), 1986.

- LÓPEZ GORGÉ, Jacinto: “Dos revistas hispano-marroquíes”. En Encuentros literarios: Marruecos-España-Iberoamérica. Volumen preparado por Mohammad Chakor. Ed. CantArabia, Madrid, 1987.

- 'ABDEL 'AAL SALEH: La idea del Islam y de la realidad social árabe-islámica en el pensamiento español contemporáneo. Tesis doctoral leída en la Facultad de Filosofía y Letras de la Universidad Autónoma de Madrid, bajo la dirección del Prof. Dr. Miguel Cruz Hernández (Inédita), 1987.

- ALEIXANDRE, Vicente: Epistolario. Selección, prólogo y notas de José Luis Cano (Ed. Alianza Tres).

- GARULO, Teresa: Bibliografía provisional de obras árabes traducidas al español (18001987). En Cuadernos de la Biblioteca Islámica "Félix María Pareja", n 11. Instituto Hispano-árabe de Cultura, Madrid, 1988.

- AHMAD 'ABD AL-'AZiZ: Al-Andalus fi-l-šic r al-isbānī bacd al-harb al-ahliyya. El Cairo, 1989.

\section{AÑOS 90:}

- MUHAMMAD SABBAG: Del fuego y de la luna y de otros poemas. (Antología). Selección, prólogo y notas de J. López Gorgé. Ed. Rialp, Colección Adonais, n 474, Madrid, 1990.

- HASAN AL-WARAGLİ: Nazarāât fī-l-adab al-magribīal-hadị. Ed. 'Ukaz, Rabat, 1990.

- LÓPEZ GORGÉ, Jacinto: Marruecos en la poesía contemporánea. Ed. Ubago, Colección Ibermagrib, Granada.

- LÓPEZ GORGÉ, Jacinto: "Memoria personal. Revistas y publicaciones literarias en el Marruecos español”. Puertaoscura (Málaga), n 3-4, s.d., pp. 62-65. 
La revista AL-MOTAMID.Verso y Prosa ${ }^{12}$ publicó los siguientes números:

En Larache: 1 (marzo 1947), 2 (abril 1947), 3 (mayo 1947), 4 (julio 1947), 5 (julio 1947), 6 (agosto 1947), 7 (septiembre 1947), 8 (octubre 1947), 9 (noviembre 1947), 10 (diciembre 1947), 11 (enero 1948), 12 (febrero 1948), 13 (marzo 1948), 14 (abril 1948), 15 (mayo 1948), 16 (1949), 17 (junio 1949), 18 (julio 1949), 19 (noviembre 1949), 20 (abril 1950), 21 (julio 1950), 22 (septiembre 1950), 23 (junio 1951), 24 (julio 1952).

En Tetuán: 25 (marzo 1953), 26 (agosto 1953), 27 (febrero 1954), 28 (septiembre 1954), 29 (octubre 1954), 30 (noviembre-diciembre, 1954), 31 (abril-junio 1955), 32 (octubrediciembre 1955), 33 (enero-marzo 1956).

${ }^{12}$ En 1951 Trina Mercader residió, por algún tiempo, en la antigua Villa Sanjurjo. El número 24 de Al-Motamid así lo reflejo en el índice de la misma, aunque en la portada seguía figurando la ciudad de Larache. Este trabajo, que hoy publicamos en homenaje a la profesora y querida amiga Eugenia Gálvez, fue presentado en el coloqio sobre "Marruecos en la cultura española contemporánea", Tetuán, Universidad Abdelmalek Essdai, 12-14 de noviembre de 1992. La Nueva antología de relatos marroquíes, de Jacinto López Gorgé, publicada en el momento de redactarse estas líneas, a finales de 1999, según me comunica amablemente el propio antólogo, incluye, entre otros autores, a Trina Mercader y su relato "Una calle del barrio moro de Larache".

Nota final: Agradezco la ayuda y, especialmente, los testimonios que me han ofrecido Muhammad Ibn ${ }^{\mathrm{c} A z u z}$, Joaquina Albarracín, Soledad Gibert, Conchita Castillo, Estrella Pérez de Amar, Miguel Cruz Hernández, Mariano Arribas, Jacinto López Gorgé, Pedro Martínez Montávez, Fernando Díaz Esteban, Leonor Martínez, y los ya fallecidos Carmen Conde, Fernando de la Granja y Guillermo Gozalbes. Y a la Biblioteca Islámica "Félix María Pareja" de la Agencia Española de Cooperación Internacional por la consulta de la bibliografía necesaria. 